\title{
The Human Leukocyte Antigen (HLA) DRB1*13:02 Allele Protects against Dementia in Continental Western Europe
}

\author{
Lisa M. James ${ }^{1,2,3,4}$, Apostolos P. Georgopoulos 1,2,3,4,5*
}

'Brain Sciences Center, Department of Veterans Affairs Health Care System, Minneapolis, MN, 55417, USA

${ }^{2}$ Department of Neuroscience, University of Minnesota Medical School, Minneapolis, MN 55455, USA

${ }^{3}$ Department of Psychiatry, University of Minnesota Medical School, Minneapolis, MN 55455, USA

${ }^{4}$ Center for Cognitive Sciences, University of Minnesota, Minneapolis, MN 55455, USA

${ }^{5}$ Department of Neurology, University of Minnesota Medical School, Minneapolis, MN 55455, USA

Article Info

\section{Article Notes}

Received: August 02, 2019

Accepted: September 09, 2019

\section{*Correspondence:}

Dr. Apostolos P. Georgopoulos, Brain Sciences Center (11B),

Minneapolis VAHCS, One Veterans Drive, Minneapolis, MN

55417, USA; Email: omega@umn.edu.

C 2019 Georgopoulos AP. This article is distributed under the terms of the Creative Commons Attribution 4.0 International License

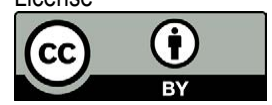

Keywords:

Human Leukocyte Antigen

Dementia

Apolipoprotein E

Persistent Antigens
Abstract

Human Leukocyte Antigen (HLA) Class II DRB1*13 alleles have recently been found to protect against age-related brain deterioration, even in the presence of apolipoprotein E4 (apoE4), ${ }^{1,2}$ suggesting a possible protection against dementia. Here we evaluated the association between the population frequency of common DRB1*13 alleles and the prevalence of dementia in Continental Western Europe. Prevalence of dementia in Continental Western Europe was derived from published reports on dementia frequency from the Global Burden of Disease Study 2016 and population totals obtained from the Population Reference Bureau. DRB1*13:01 and DRB1*13:02 allele frequencies were obtained from a publicly available database (allelefrequency.net) and apolipoprotein $\mathrm{E}$ was obtained from published reports on the world distribution of apoE4. The prevalence of dementia in 14 Continental Western European (CWE) countries, where life expectancy is practically identical, significantly decreases exponentially with increasing frequency of DRB1*13:02 $\left(R^{2}=\right.$ $0.452, P=0.008)$, even when adjusted for the prevalence of apolipoprotein E4 allele, a known risk factor for Alzheimer's disease. This finding documents the protective effect of DRB1*13:02 on dementia prevalence in CWE. Since the function of HLA class II genes is to aid in the elimination of pathogens by enabling the production of antibodies against their antigens in specific immunity, the protective effect of DRB1*13:02 points to the presence of persistent harmful antigens as causal factors in development of dementia, antigens specific to DRB1*13:02 that could not be eliminated in its absence.

\section{Introduction}

Dementia is now the $5^{\text {th }}$ leading cause of death, representing a significant global health problem ${ }^{3}$. Over 50 million people worldwide are living with dementia, and the number of affected individuals is expected to increase significantly over the next 30 years as the population ages. Western Europe has the largest number of people living with dementia ${ }^{4,5}$ and has consistently been found to have one of the highest prevalence rates of dementia for individuals 60 years old and above ${ }^{5,6}$. Within Western Europe, rates of dementia have been shown to vary substantially by country ${ }^{7-9}$. Recent studies have documented reduced incidence of dementia across Western Europe which has been largely attributed to improved health and educational attainment ${ }^{10}$; however, such modifiable risk factors are estimated to account for only $35 \%$ of dementia risk ${ }^{11}$, highlighting the sustained influence of genetic factors on this risk. A well-established genetic factor is the apolipoprotein E4 (apoE4) allele, with dementia risk increasing in a dose-dependent manner ${ }^{12}$. However, several recent genome-wide association studies have implicated numerous other single nucleotide polymorphisms 
(SNPs) in dementia ${ }^{13-15}$, supporting the role of genetic influences on dementia that extend beyond apoE4.

\section{Human Leukocyte Antigen (HLA)}

Recent evidence suggests that HLA genes, which play a critical in specific immunity, may also influence risk for dementia ${ }^{13-16}$. Located in the Major Histocompatibility Complex (MHC) of chromosome 6, HLA genes code for glycoproteins that reside on the surface of most cells and facilitate elimination of foreign antigens. Two main classes of HLA genes, namely Class I (A, B, C) and Class II (DRB, DQB, $\mathrm{DPB}$ ), facilitate immune protection from harmful antigens via presentation of endogenous antigenic peptides to CD8 receptors to signal cell destruction (Class I) or exogenous antigenic peptides to CD4 receptors to promote antibody production (Class II). Successful elimination of foreign antigens necessitates a match between one's HLA genes and epitopes of those antigens; the absence of a match may result in persistent antigens that may, in turn, lead to neuronal damage ${ }^{16,17}$. Although each person has a limited repertoire of HLA-coded proteins based on Mendelian inheritance (two alleles of each major Class I and Class II gene), resistance to foreign antigens is maximized at the population level via allelic diversity ${ }^{18,19}$. Indeed, HLA genes are the most highly polymorphic in the human genome, and vary by region and ethnicity to promote elimination of local foreign antigens; yet, some alleles are more common than others ${ }^{20,21}$, presumably conferring protection against common antigens such as viruses and bacteria.

\section{Infections and Dementia}

Numerous infectious viruses and bacteria including herpes viruses, Helicobacter pylori, Chlamydophila pneumoniae, and Borrelia burgdorfer, among others, have been implicated in dementia $^{22}$. In fact, a prominent theory of dementia posits that reactivation of latent herpes simplex virus type 1 (HSV1), particularly among apoE4 carriers, causes cumulative damage that ultimately results in Alzheimer's dementia (AD) ${ }^{23}$. To date, no single infectious cause of dementia has been conclusively identified but the evidence linking such pathogens to dementia is substantial ${ }^{22}$. Specifically, (1) various pathogens have been detected in brain tissues of individuals with $\mathrm{AD},(2)$ chronic infections are associated with neurodegeneration, neurofibrillary tangles, and $\beta$-amyloid plaques, histopathological features that are considered to be the hallmarks of $\mathrm{AD}$, and (3) neuroinflammation is common to many infectious diseases and is also characteristic of AD. Thus, it seems likely that infectious agents are associated with dementia; however, other factors including genetic composition, may moderate the association.

\section{Persistent Antigens}

We have proposed that HLA composition is a critical factor in disease outcomes, including dementia, following exposure to foreign antigens ${ }^{17}$. That is, if one's HLA composition is a match for a specific antigen, the immune system is able to eliminate harmful effects of the antigen through cell destruction and/or antibody production. However, in the absence of an HLA-antigen match, the antigen may not be sufficiently eliminated, resulting in deleterious effects including inflammation, cell damage, and ultimately neurodegeneration. These harmful effects are hypothesized to be exacerbated in apoE4 carriers $^{16}$. One function of apoE is neuronal repair; that is, apoE is synthesized in response to neural damage to facilitate repair. However, apoE4 is relatively less stable than other isoforms, resulting in neurotoxic fragments ${ }^{24}$; consequently, the effect of apoE synthesis in E4 carriers may cause additional secondary neuronal damage ${ }^{16}$. In contrast, no secondary damage is expected in apoE2 or apoE3 carriers. Thus, HLA composition is hypothesized to play a primary role in disease outcomes following exposure to foreign antigens with apoE playing a secondary role ${ }^{16}$.

\section{HLA Protection Against Neurodegeneration}

Mounting evidence implicates HLA protection against various disease outcomes. For example, DRB1*13:01 and DRB1*13:02, which differ by a single amino acid and are among the most common alleles of the Class II DRB gene $^{21}$, have been shown to confer protection against various autoimmune diseases ${ }^{25,26}$, many of which have effects on the brain. DRB1*13:02 has also been shown to protect not only against the occurrence of Gulf War Illness, ${ }^{27}$ a neuroimmune condition affecting veterans of the Persian Gulf $\mathrm{War}^{28}$, but also against GWI-related brain atrophy ${ }^{29}$ that is characteristic of the illness ${ }^{30}$. Finally, we have recently shown protective effects of DRB $1 * 13$ alleles against age-related brain atrophy ${ }^{1}$ and functional deterioration $^{2}$ in cognitively healthy women, even after accounting for presence of apoE4. That is, age-related brain atrophy and increasing neural network variability were observed in women without protective DRB1*13 alleles; no such age-related effects were found in those with the protective allele. Taken together, common DRB1*13 alleles confer protection against various conditions affecting the brain, including age-related brain deterioration, leading us to hypothesize that HLA DRB1*13:02 and DRB1*13:01 alleles may also protect against dementia ${ }^{16}$.

\section{The Present Study}

Here we tested the hypothesis that common HLA DRB1*13 alleles protect against dementia using a population-based, genetic-epidemiological approach. We assessed the association of the frequency of those alleles and prevalence of dementia in 14 countries of Continental Western Europe (CWE), namely Austria, Belgium, Denmark, Finland, France, Germany, Greece, Italy, Netherlands, Norway, Portugal, Spain, Sweden, and Switzerland. 


\section{Methods}

For each of the $14 \mathrm{CWE}$ countries, we obtained estimates of prevalence of dementia, frequency of HLA DRB1*13:02 and 13:01, prevalence of apoE4, and life expectancy as follows. Dementia prevalence was computed by dividing the number of people with dementia in 2016 in each country ${ }^{4}$ by the total population of the country in $2016^{31}$ and expressed as a percentage. We also tabulated the life expectancy ${ }^{31}$. These data are shown in Table 1. (b) The frequencies of DRB1*13:02 and DRB1*13:01 alleles were obtained on March 3, 2019 by querying the Allele*Frequencies database (http://www.allelefrequencies.net) . We calculated the average frequency of DRB1*13:02 and DRB1*13:01 across different studies within each country using high-resolution (4-digit) typing; the average frequency of each allele per country and sample size is shown in Table 2. Finally, (c) the apoE4 prevalence was obtained from Singh et al. ${ }^{32}$ and shown in Table 3.

\section{Statistical Analyses}

Standard statistical analyses were performed including calculating univariate descriptive statistics (mean, standard deviation, coefficient of variation) and regression and correlation analyses between dementia prevalence and allele frequency. All statistical analyses were conducted using the IBM-SPSS statistical package (version 23).

\section{Results}

\section{Life expectancy}

Life expectancy was practically identical in the $14 \mathrm{CWE}$ countries (mean $\pm \mathrm{SD}$, coefficient of variation): $79.1 \pm 1.1$

Table 1. Dementia prevalence and life expectancy for countries in continental Western Europe.

\begin{tabular}{|c|c|c|c|c|c|c|}
\hline Country & N Dementia ${ }^{a}$ & $\stackrel{\mathbf{N}}{\text { total population }^{\mathrm{b}}}$ & $\begin{array}{l}\text { Dementia } \\
\text { Prevalence }\end{array}$ & $\begin{array}{l}\text { Life expectancy } \\
\text { women }^{b}\end{array}$ & $\begin{array}{l}\text { Life expectancy } \\
\text { men }^{\mathrm{b}}\end{array}$ & $\begin{array}{l}\text { Average life } \\
\text { expectancy }\end{array}$ \\
\hline Austria & 126914 & 8800000 & 1.442 & 84 & 79 & 81.5 \\
\hline Belgium & 181350 & 11300000 & 1.605 & 84 & 79 & 81.5 \\
\hline Denmark & 55336 & 5700000 & 0.971 & 82 & 79 & 80.5 \\
\hline Finland & 83950 & 5500000 & 1.526 & 85 & 78 & 81.5 \\
\hline France & 877760 & 64600000 & 1.359 & 85 & 79 & 82 \\
\hline Germany & 1201668 & 82600000 & 1.455 & 83 & 78 & 80.5 \\
\hline Greece & 192563 & 10800000 & 1.783 & 84 & 78 & 81 \\
\hline Italy & 1370308 & 60600000 & 2.261 & 85 & 80 & 82.5 \\
\hline Netherlands & 192425 & 17000000 & 1.132 & 83 & 80 & 81.5 \\
\hline Norway & 67207 & 5200000 & 1.292 & 84 & 80 & 82 \\
\hline Portugal & 166660 & 10300000 & 1.618 & 83 & 77 & 80 \\
\hline Spain & 830915 & 43300000 & 1.919 & 85 & 80 & 82.5 \\
\hline Sweden & 142735 & 9900000 & 1.442 & 84 & 80 & 82 \\
\hline Switzerland & 115476 & 8400000 & 1.375 & 85 & 81 & 83 \\
\hline
\end{tabular}

${ }^{a}$ Obtained from Nichols et al., 2019. ${ }^{4 b}$ Obtained from Population Reference Bureau, $2016^{31}$

Table 2. HLA DRB1*13:01 allele frequency per country in continental Western Europe. ${ }^{\mathrm{a}}$

\begin{tabular}{|l|c|c|c|}
\hline \multicolumn{1}{|c|}{ Country } & Sample size (2n) & $\begin{array}{c}\text { DRB1*13:02 } \\
\text { frequency }\end{array}$ & $\begin{array}{c}\text { DRB1*13:01 } \\
\text { frequency }\end{array}$ \\
\hline Austria & 400 & 0.062 & 0.062 \\
\hline Belgium & 198 & 0.035 & 0.076 \\
\hline Denmark & 110 & 0.056 & 0.083 \\
\hline Finland & 482 & 0.025 & 0.087 \\
\hline France & 1260 & 0.047 & 0.058 \\
\hline Germany & 140300 & 0.043 & 0.067 \\
\hline Greece & 1848 & 0.033 & 0.035 \\
\hline Italy & 12562 & 0.038 & 0.051 \\
\hline Netherlands & 3632 & 0.062 & 0.072 \\
\hline Norway & 362 & 0.050 & 0.080 \\
\hline Portugal & 550 & 0.036 & 0.084 \\
\hline Spain & 3850 & 0.033 & 0.076 \\
\hline Sweden & 568 & 0.038 & 0.072 \\
\hline Switzerland & 41664 & 0.053 & 0.067 \\
\hline
\end{tabular}

a. Obtained from allelefrequencies.net March 3, 2019.
Table 3. ApoE4 prevalence per country in continental Western Europe. $^{\mathrm{a}}$

\begin{tabular}{|l|c|c|}
\hline \multicolumn{1}{|c|}{ Country } & Sample size & ApoE4 prevalence \\
\hline Austria & 683 & 0.105 \\
\hline Belgium & 1660 & 0.128 \\
\hline Denmark & 1620 & 0.127 \\
\hline Finland & 7285 & 0.215 \\
\hline France & 8247 & 0.114 \\
\hline Germany & 6123 & 0.132 \\
\hline Greece & 216 & 0.065 \\
\hline Italy & 4189 & 0.083 \\
\hline Netherlands & 5484 & 0.154 \\
\hline Norway & 1097 & 0.174 \\
\hline Portugal & 381 & 0.169 \\
\hline Spain & 4863 & 0.094 \\
\hline Sweden & 686 & 0.205 \\
\hline Switzerland & 2061 & 0.109 \\
\hline
\end{tabular}

a. Obtained from Singh et al., $2006^{32}$. 
$\mathrm{y}, 1.4 \%$ (men), $84.0 \pm 0.96 \mathrm{y}, 1.1 \%$ (women), $81.6 \pm 0.14 \mathrm{y}$ $1.0 \%$ (average between men and women).

\section{Effect of DRB1*13:02 on dementia prevalence}

The prevalence of dementia decreased exponentially with increasing frequency of DRB1*13:02 (Figure 1A-B), according to the following regression equation:

$$
\text { Dementia Prevalence }(\%)=2.529 e^{-12.275 f_{1802}}
$$

where $f$ is the frequency of DRB1*13:02. This model was highly statistically significant $(\mathrm{P}=0.008)$ and explained $45.2 \%$ of the variance $\left(R^{2}=0.452\right)$. Figure 2 illustrates graphically the substantial magnitude of this protective effect: doubling the DRB1*13:02 frequency (from 0.03 to 0.06 ) is associated with $31 \%$ reduction of dementia prevalence (from $1.75 \%$ to $1.21 \%$ ).

By taking the natural logarithms (ln) on both sides, we get, equivalently,

$\ln \left(\right.$ Dementia Prevalence \%) $=\operatorname{In}(2.529)-12.275 f_{1302}=$ $0.928-12.275 f_{1302}$

This effect was robust (Spearman's rank correlation $=-0.750, \mathrm{P}=0.002$ ). These findings indicate a protective role of the DRB1:13:02 allele for dementia. In addition, this effects
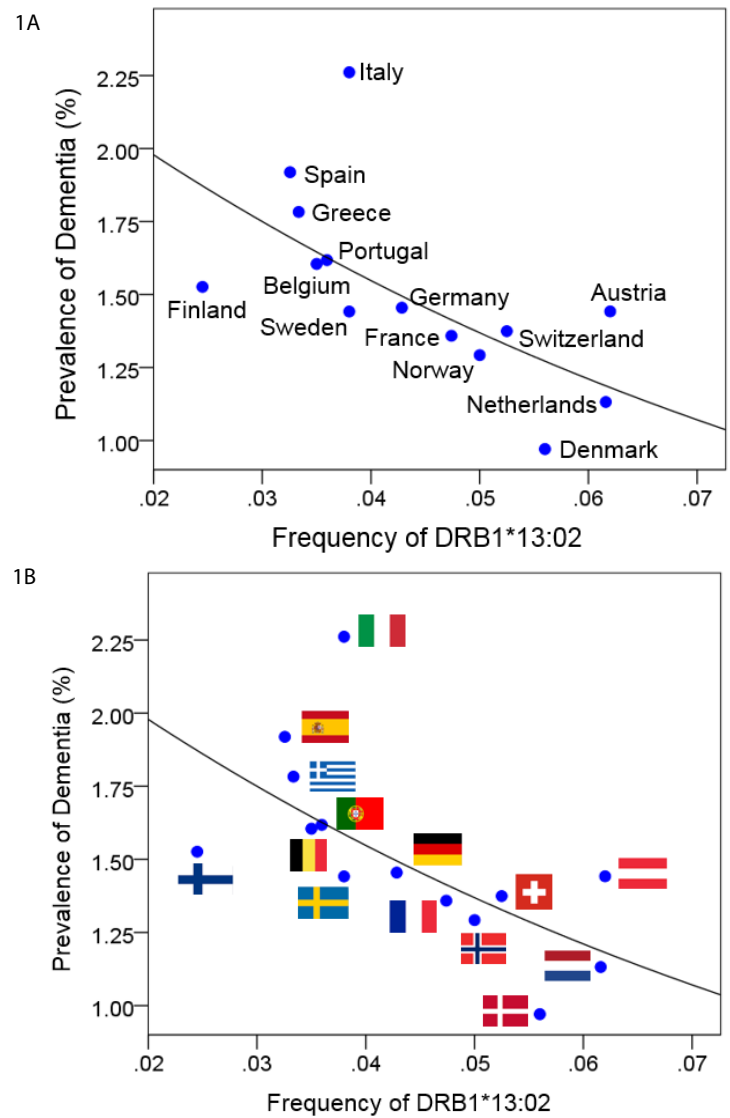

Figure 1: Dementia prevalence (5) is plotted against DRB1*13:02 frequency with countries identified by names $(A)$ or national flags (B). (See text for details.)

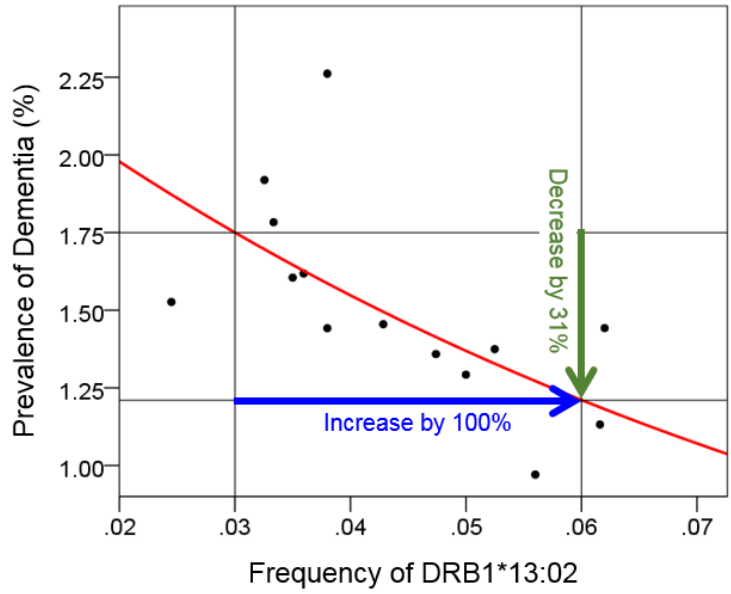

Figure 2: Illustration of the quantitative effect of DRB1*13:02 frequency on dementia prevalence, based on the fitted exponential fit of equation 2 (see text). An increase of $100 \%$ (doubling) of the DRB1*13:02 frequency results in $31 \%$ reduction in dementia prevalence.

was independent of apoE4 prevalence since it was still present and highly significant when the effect of apoE4 prevalence was partialed out; specifically, the partial correlation of dementia prevalence vs DRB1*13:02 frequency, accounting for apoE4 prevalence, was $-0.782, \mathrm{P}=0.002$ ).

\section{Effect of DRB1*13:01 on dementia prevalence}

Dementia prevalence decreased with increasing frequency of DRB1*13:01 but this relation did not reach statistical significance $\left(\mathrm{R}^{2}=0.157, \mathrm{P}=0.161\right.$; exponential fit); the partial correlation of dementia prevalence vs. DRB1*13:01 frequency, accounting for apoE4 prevalence, was $-0.218, \mathrm{P}=0.474$ ).

\section{Discussion}

These results extend prior findings demonstrating protective effects of HLA DRB1*13:02 on brain structure ${ }^{1}$ and function ${ }^{2}$ in healthy individuals to suggest DRB1*13:02 protects against dementia at the population level. The mechanisms through which HLA DRB1*13:02 confers protection against dementia remain to be elucidated; however, the primary role of HLA DRB1*13:02 (and other HLA Class II alleles) in elimination of foreign antigens via antibody production suggests that host protection from foreign antigens plays an integral role. While elimination of foreign antigens appears to protect against dementia, the persistent antigen hypothesis ${ }^{17}$ posits that inability to eliminate foreign antigens results in inflammation and neural damage that may ultimately result in neurodegeneration and dementia. DRB1*13:02 and DRB1*13:01 differ by a single amino acid; yet that small difference alters the electrostatic properties of the binding groove ${ }^{33}$ which is reflected in differential ability to bind to exogenous antigenic peptides as a first step in antibody production. Such binding differences are likely 
associated with the current finding that DRB1*13:02 but not DRB1*13:01 was highly significantly associated with protection from dementia. In light of the present findings, the antigens to which HLA DRB1*13:02 bind take on new relevance in terms of protection against dementia. A search of human studies in the immune epitope database (IEDB; retrieved March 4, 2019) indicates that 1820 epitopes from 153 antigens bind to HLA-DRB1*13:02, including several from viruses such as hepatitis B and C, HIV, several types of herpes virus, influenza, mycobacterium tuberculosis, and malaria, among others. That is, DRB1*13:02 carriers are equipped to create antibodies to facilitate elimination of these antigens; in the absence of a match, the antigens may persist and create negative downstream effects including dementia.

Given the widely recognized association between apoE4 and dementia risk, the current findings that DRB1*13:02 prevalence is negatively associated with dementia prevalence, independently of apoE4, is particularly noteworthy and consistent with our prior findings that DRB1*13:02 protects against age-related deterioration in brain function in healthy women, regardless of apoE4 genotype. ${ }^{2}$ Taken together, these findings suggest that apoE4 effects are secondary to HLA ${ }^{34}$. To that end, we have recently posited that the risk associated with apoE4 status is negated given an HLA-antigen match ${ }^{16}$. In contrast, in the absence of an HLA-antigen match, persistent antigens cause neural damage which, in turn, activates synthesis of apoE to facilitate neuronal repair ${ }^{24}$. Whereas apoE2 and apoE3 may enable neuronal repair, neurotoxic apoE4 fragments may cause additional neuronal damage. That perspective, together with the present findings, shines the spotlight on HLA as playing a fundamental role in preventing dementia. As such, we would expect HLA DRB1*13:02 to be scarce among individuals with dementia.

\section{Limitations}

The main limitation of this study is that in some countries apoE and DRB1*13 allele frequencies were derived from relatively small samples; we assume the frequencies accurately reflect the population prevalence. In addition, the present study focused exclusively on DRB1*13:01 and DRB1*13:02 based on our prior work demonstrating protective effects of those alleles against brain deterioration in healthy women. The present results do not preclude the possibility of other protective HLA alleles; indeed, studies evaluating the association of other HLA alleles with dementia prevalence are underway.

\section{Conclusions}

Our results suggest that DRB $1 * 13: 02$ protects against dementia, implicating adaptive immunity and elimination of foreign antigens in dementia prevention. Future studies aimed at identifying and eliminating persistent harmful antigens that contribute to dementia may prove useful in reducing the global burden of dementia.

\section{Acknowledgments}

Partial funding was provided by the University of Minnesota (the Kunin Professorship for Women's Healthy Brain Aging, the McKnight Presidential Chair of Cognitive Neuroscience, and the American Legion Brain Sciences Chair) and the U.S. Department of Veterans Affairs. The sponsors had no role in the current study design, analysis or interpretation, or in the writing of this paper. The contents do not represent the views of the U.S. Department of Veterans Affairs or the United States Government.

\section{References}

1. James LM, Christova P, Lewis SM, et al. Protective effect of human leukocyte antigen (HLA) allele DRB1*13: 02 on age-related brain gray matter volume reduction in healthy women. EBioMedicine. 2018; 29: 31-37. doi:10.1016/j.ebiom.2018.02.005.

2. James LM, Dolan S, Leuthold AC, et al. The effects of human leukocyte antigen DRB1* 13 and apolipoprotein E on age-related variability of synchronous neural interactions in healthy women. EBioMedicine. 2018; 35: 288-94. doi:10.1016/j.ebiom.2018.08.026.

3. World Health Organization, (WHO), Global Health Estimates 2016: Deaths by Cause, Age, Sex, by Country and by Region, 2000-2016. WHO, 2018. https://www.who.int/healthinfo/global_burden_ disease/estimates/en/index1.html. Accessed December 7, 2018.

4. Nichols E, Szoeke CEI, Vollset SE, et al. Global, regional, and national burden of Alzheimer's disease and other dementias, 1990-2016: a systematic analysis for the Global Burden of Disease Study 2016. Lancet Neurol. 2019; 18: 88-106. doi:10.1016/S1474-4422(18)30403-4

5. Prince M, Bryce R, Albanese E, et al. The global prevalence of dementia: a systematic review and meta-analysis. Alzheimers Dement. 2013; 9: 63-75. doi:10.1016/j.jalz.2012.11.007

6. Ferri CP, Prince M, Brayne C, et al. Global prevalence of dementia: A Delphi consensus study. Lancet. 2005; 366(9503): 2112-7. doi:10.1016/S0140-6736(05)67889-0.

7. Berr C, Wancata J, Ritchie K. Prevalence of dementia in the elderly in Europe. Eur. Neuropsychopharm. 2005; 15: 463-71. doi:10.1016/j. euroneuro.2005.04.003

8. Lobo A, Launer LJ, Fratiglioni L, et al. Prevalence of dementia and major subtypes in Europe: a collaborative study of population-based cohorts. Neurology. 2000; 54(11, Supplement 5): S4-S9.

9. Niu H I, Álvarez-Álvarez F, Guillén-Grima I. Aguinaga-Ontoso. Prevalence and incidence of Alzheimer's disease in Europe: A meta-analysis. Neurología. 2017; 32(8): 523-532. doi:10.1016/j. nrleng.2016.02.009

10. Wu YT, Beiser AS, Breteler MM, et al. The changing prevalence and incidence of dementia over time-current evidence. Nat Rev Neurol 2017; 13(6): 327-339. doi:10.1038/nrneurol.2017.63

11. Livingston G, Sommerland A, Orgeta V, et al. Dementia prevention, intervention, and care. Lancet. 2017; 390(10113): 2673-2734. doi:10.1016/S0140-6736(17)31363-6

12. Corder EH, Saunders AM, Strittmatter WJ, et al. Gene dose of apolipoprotein E type 4 allele and the risk of Alzheimer's disease in late onset families. Science. 1993; 261(5123): 921-3.

13. Desikan RS, Fan CC, Wang Y, et al. Personalized genetic assessment of age-associated Alzheimer's disease risk: Development and validation of a polygenic hazard score. PLoS Med. 2017; 14: e1002258. 


\section{doi:10.1371/journal.pmed.1002258}

14. Lambert JC, Ibrahim-Verbaas CA, Harold D, et al. Meta-analysis of 74,046 individuals identifies 11 new susceptibility loci for Alzheimer's disease. Nat Genet. 2013; 45(12): 1452-1458. doi:10.1038/ng.2802

15. Steele NZ, Carr JS, Bonham LW, et al. Fine-mapping of the human leukocyte antigen locus as a risk factor for Alzheimer disease: a case- control study. PLOS Med. 2017; 14(3): e1002272. doi:10.1371/ journal.pmed.1002272.

16. James LM, Georgopoulos AP. Human leukocyte antigen as a key factor in preventing dementia and associated apolipoprotein E4 risk. [published online April 12, 2019]. Front Aging Neurosci. doi:10.3389/ fnagi.2019.00082.

17. James LM, Georgopoulos AP. Persistent antigens hypothesis: The human leukocyte antigen (HLA) connection. J Neurol Neuromed. 2018; 3(6): 27-31. doi:10.29245/2572.942X/2018/6.1235

18. Bergström TF, Josefsson A, Erlich HA, et al. Recent origin of HLA-DRB1 alleles and implications for human evolution. Nat Genet. 1998; 18 237.

19. Buhler S, Sanchez-Mazas A. HLA DNA sequence variation among human populations: molecular signatures of demographic and selective events. PloS One. 2011; 6(2): e14643. doi:10.1371/journal. pone. 0014643 .

20. Mack SJ, Cano P, Hollenbach JA, et al. Common and well-documented HLA alleles: 2012 update to the CWD catalogue. Tissue Antigens. 2013; 81(4): 194-203. doi:10.1111/tan.12093.

21. Maiers M, Gragert L, Klitz W. High resolution HLA alleles and haplotypes in the US population. Human Immunol. 2007; 68: 779788. doi:10.1016/j.humimm.2007.04.005
22. Mawanda F, Wallace R. Can infections cause Alzheimer's disease? Epidemiol. Rev. 2013 ; 35: 161-80. doi:10.1093/epirev/mxs007

23. Itzhaki RF. Corroboration of a major role for herpes simplex virus type 1 in alzheimer's disease. Front. Aging Neurosci. 2018; 10: e00324. doi: 10.3389/fnagi.2018.00324

24. Mahley RW, Huang Y. Apolipoprotein E sets the stage: Response to injury triggers neuropathology. Neuron. 2012; 76: 871-885. doi:10.1016/j.neuron.2012.11.020.

25. Bettencourt A, Carvalho C, Leal B, et al. The protective role of HLADRB1*13 in autoimmune dis eases. J Immunol Res. 2015; 2015; e948723. doi:10.1155/2015/948723.

26. Furukawa H, Oka S, Tsuchiya N, et al. The role of common protective alleles HLA-DRB1* 13 among systemic autoimmune diseases. Genes Immun. 2017; 18(1): 1-7. doi:10.1038/gene.2016.40

27. Georgopoulos AP, James LM, Mahan MY, et al. Reduced human leukocyte antigen (HLA) protection in Gulf War Illness (GWI). EBioMedicine. 2016; 3: 79-85. doi:10.1016/j.ebiom.2015.11.037

28. Georgopoulos AP, James LM, Carpenter AF, et al. Gulf War illness (GWI) as a neuroimmune disease. Exp Brain Res. 2017; 235(10): 3217-3225. doi:10.1007/s00221-017-5050-0.

29. James LM, Christova P, Engdahl BE, et al. Human leukocyte antigen (HLA) and Gulf War Illness (GWI): HLA-DRB1*13:02 spares subcortical atrophy in Gulf War veterans. EBioMedicine. 2017; 26: 126-131. doi: 10.1016/j.ebiom.2017.11.005

30. Christova P, James LM, Engdahl BE, et al. Subcortical brain atrophy in Gulf War Illness. Exp Brain Res. 2017; 235: 2777-2786. doi: 10.1007/ s00221-017-5010-8

31. Population Reference Bureau. 2016 world population data sheet with a special focus on human needs and sustainable resources. Population Reference Bureau, Washington, DC, 2016. https://www. prb.org/2016-world-population-data-sheet/. Accessed February 5, 2019.

32. Singh PP, Singh M, Mastana SS. APOE distribution in world populations with new data from India and the UK. Ann Hum Biol. 2006; 33(3): 279-308. doi:10.1080/03014460600594513

33. Hov R, Kosmoliaptsis V, Traherne JA, et al. Electrostatic modifications of the HLA-DR P9 peptide-binding pocket and susceptibility to primary sclerosing cholangitis. Hepatol. 2011; 53(6): 1967-1976. doi:10.1002/hep.24299.

34. Savaki H. A specific immunity brain aging gene with a future. EBioMedicine. 2018; 36: 9. doi:10.1016/j.ebiom.2018.08.044 